\title{
Prediction and analysis of the modular structure of cytochrome P450 monooxygenases
}

\author{
Demet Sirim, Michael Widmann, Florian Wagner, Jürgen Pleiss
}

\begin{abstract}
Background: Cytochrome P450 monooxygenases (CYPs) form a vast and diverse family of highly variable sequences. They catalyze a wide variety of oxidative reactions and are therefore of great relevance in drug development and biotechnological applications. Despite their differences in sequence and substrate specificity, the structures of CYPs are highly similar. Although being in research focus for years, factors mediating selectivity and activity remain vague.

Description: This systematic comparison of CYPs based on the Cytochrome P450 Engineering Database (CYPED) involved sequence and structure analysis of more than 8000 sequences. 31 structures have been applied to generate a reliable structure-based HMM profile in order to predict structurally conserved regions. Therefore, it was possible to automatically transfer these modules on CYP sequences without any secondary structure information, to analyze substrate interacting residues and to compare interaction sites with redox partners.

Conclusions: Functionally relevant structural sites of CYPs were predicted. Regions involved in substrate binding were analyzed in all sequences among the CYPED. For all CYPs that require a reductase, two reductase interaction sites were identified and classified according to their length. The newly gained insights promise an improvement of engineered enzyme properties for potential biotechnological application. The annotated sequences are accessible on the current version of the CYPED. The prediction tool can be applied to any CYP sequence via the web interface at http://www.cyped.uni-stuttgart.de/cgi-bin/strpred/dosecpred.pl.
\end{abstract}

\section{Background}

Cytochrome P450 monooxygenases (CYPs) are a ubiquitous protein family, existing in all eukaryotes, most prokaryotes and Archae. These heme-containing enzymes catalyze the monooxygenation of a large variety of substrates [1]. CYPs have an essential function in drug metabolism, hence focussed in the pharmaceutical industry [2]. Besides, they are of great interest for synthetical application in biotechnology as versatile biocatalysts [3]. A profound knowledge in the factors mediating selectivity and activity of these proteins is a prerequisite in the development of CYPs with improved properties. Therefore, deeper insights in the relationships between sequence, structure and function are of great interest.

According to Nelson's classification [4] CYPs are grouped into homologous families and superfamilies, predominantly based on sequence similarity. The sequence

\footnotetext{
* Correspondence: juergen.pleiss@itb.uni-stuttgart.de
Institute of Technical Biochemistry, University of Stuttgart, Allmandring 31,

* Correspondence: juergen.pleiss@itb.uni-stuttgart.de
Institute of Technical Biochemistry, University of Stuttgart, Allmandring 31, 70569 Stuttgart, Germany
}

(c) 2010 Sirim et al; licensee BioMed Central Ltd. This is an Open Access article distributed under the terms of the Creative Commons Attribution License (http://creativecommons.org/licenses/by/2.0), which permits unrestricted use, distribution, and reproduction in any medium, provided the original work is properly cited. identity between proteins from different superfamilies is amino acids are totally conserved, the glutamic acid and the arginine of the ExxR-motif, which is involved in stabilizing the core and heme-binding [6], and the heme-binding cysteine. However, the increasing number of crystal structures shows that despite this unusual variability the overall structure is highly conserved: CYPs consists of structural conserved modules that are essential for structure and function, and of variable regions that mediate the individual biochemical properties. The defined conserved secondary structures are named $\alpha \mathrm{A}-\mathrm{L}$ and $\beta 1-5$ and could be identified in all CYP structures and make up the so called CYP-fold [7-9].

Most CYPs require interaction with a reductase to provide electrons, either as separate proteins or as fusion proteins. Depending on the nature of their electron transfer partner, CYPs are assigned to different classes. Although, no consensus has been reached in the definition of this classification, there are several proposed 
schemes which subdivide CYPs in up to nine classes [10-12]. The most general one, which was applied in this work, discriminates between two major classes of CYPs [13]: class I, which comprises mitochondrial and bacterial CYPs and class II which comprises CYPs interacting with a cytochrome P450 reductase-type (CPR-type) FMN/ FAD reductase and represents a simplification of the widely accepted classification scheme by Kelly et al. in [1]. Further, there are CYPs known which do not need a reductase for their reaction [14]. Fusion proteins, such as the self-sufficient class II CYP 102A1 from Bacillus megaterium ( $\mathrm{P} 450 \mathrm{BM}-3$ ) which contains a heme domain and a reductase, as well as those CYPs which do not require any reductase interaction appear very rarely in nature [15]. Therefore, in most CYPs the interaction with their appropriate redox partner is prerequisite for their reaction to occur. Many different CYP isoenzymes interact with only one reductase, and it is assumed that CYPs of the same class are comparable in regard to their reductase interaction sites [16]. It is expected that there are favorable electrostatic interactions between CYPs and their electron transfer partner [17]. A crystal structure for a CYP-reductase-complex is not yet available. Even though the kinetics in $\mathrm{P} 450$ reduction may not be generalized among different $\mathrm{P} 450$ systems, and the concepts regarding the influence of a rate-limiting step are not universal [18], the electron transfer from the reductase to the heme domain is often slow and one of the rate-limiting aspects in many CYP systems [19]. However, the interactions between the components of the electron transfer systems still remain unclear. A deeper understanding of the factors determining reductase interaction gained by the analysis of the reductase interaction sites of CYPs will assist in improving interactions and consequently lead to optimized enzymes for biocatalytic applications [20].

Previous analyses of the structure conservation in CYPs showed that all CYPs have a well-conserved heme-binding structural core formed out of $\alpha \mathrm{D}, \alpha \mathrm{E}, \alpha \mathrm{I}$, and $\alpha \mathrm{L}$ and $\alpha \mathrm{J}$ and $\alpha \mathrm{K}$ [21]. The $\beta$-bulge region which contains the thiolate heme ligand is referred to as Cyspocket. Between $\alpha \mathrm{K}$ and the Cys-pocket, a structurally conserved region is located, the so-called 'meander' loop. It is spanned by 7-10 amino acid residues and is supposed to play a role in heme binding and stabilization of the tertiary structure. The proposed reductase interaction face of CYPs mainly comprises the $\alpha \mathrm{J} / \alpha \mathrm{J}^{\prime}$ and the insertion following the meander loop [6]. Since the structures of all CYPs are highly similar, but differ in substrate specificity and their electron transfer partners, the different biochemical properties of CYPs are mediated by the diverse regions, which vary in both sequence and structure [8].
Six regions which are involved in recognition and binding of substrates and hence determine substrate specificity were described as SRSs (substrate recognition sites [22]). SRS1 lies in the highly variable loop region between $\alpha \mathrm{B}$ and $\alpha \mathrm{C}$ (BC-loop), SRS2 is located in the C-terminal end of $\alpha \mathrm{F}, \mathrm{SRS} 3$ and SRS4 are spanned by the N-terminal regions of $\alpha \mathrm{G}$ and $\alpha \mathrm{I}, \beta 1-4$ houses SRS5 and $\beta 4-1$ SRS6. While the access of the substrate to the binding pocket is limited by flexible regions in the entrance channel, such as $\alpha \mathrm{F}$ and $\alpha \mathrm{G}$ which undergo strong conformational changes upon substrate binding [23,24], the regions flanking directly the binding pocket and thus limiting the access of the substrate to the heme, namely $\alpha \mathrm{I}$, the BC-loop region and SRS5, were observed to remain rigid during simulation $[25,26]$. In a systematic analysis of SRS5 in more than 6300 sequences, single substrate- and heme-interacting residues could be identified in this region [27]: Thus, a hotspot for regio- and stereoselectivity in one residue in SRS5 and one position in the BC-loop (F87), were previously reported as key residues in determining activity, regio- and stereoselectivity in CYP102A1 [28-30]. Combinations of variants of these two positions were applied to design a minimal mutant library with improved selectivity [31]. Due to the high variability of the $\mathrm{BC}$-loop, the identification of position 87 in CYP102A1 in other CYPs, remains a challenge for sequences without structural information.

To serve as a tool for a comprehensive comparison of protein sequences and structures within the vast and diverse family of CYPs in order to transfer the newly gained insights among the CYP sequences, the Cytochrome P450 Engineering Database (CYPED) [32] has been designed. In its current version 2.02 it contains 8614 sequences [33]. The highly similar structures have been compared in detail to identify the common core and to assign the variable regions. For this purpose a structural alignment was used as a base to generate a reliable structure profile. With this profile all structurally conserved regions (SCR) could be predicted and annotated among all CYPED protein sequence entries, hence allowing a structural navigation in those sequences lacking structural information. Beyond this, the CYPED website provides an interface which allows the prediction of the SCRs for every user-specified CYP sequence.

\section{Data}

\section{CYP Structures}

A set of 31 PDB structures [34] was extracted from version 1.1 of the CYPED [32] as listed in table 1. The selection includes 16 bacterial structures of class I and 12 CYPs assigned to class II CYPs, comprising CYPs which interact with a CPR-type FMN/FAD reductase. The structures in this class are predominantly of 
Table 1 List of CYP structures analyzed in this work

\begin{tabular}{|c|c|c|c|c|}
\hline CYP & $P D B$ entry & Resolution $[\AA ̊]$ & Ligand & Organism \\
\hline \multicolumn{5}{|c|}{ Class II CYPs (CPR-type) } \\
\hline $1 \mathrm{~A} 2$ & $2 \mathrm{HI} 4$ & 1.95 & + & Homo sapiens \\
\hline $2 \mathrm{~A} 6$ & $1 Z 10$ & 1.90 & + & Homo sapiens \\
\hline $2 \mathrm{~A} 13$ & 2P85 & 2.35 & + & Homo sapiens \\
\hline 2B4 & 1SUO & 1.90 & + & Oryctolagus cuniculus \\
\hline $2 \mathrm{C} 5$ & $1 \mathrm{~N} 6 \mathrm{~B}$ & 2.30 & + & Oryctolagus cuniculus \\
\hline $2 \mathrm{C} 8$ & 1PQ2 & 2.70 & + & Homo sapiens \\
\hline $2 C 9$ & $10 G 2$ & 2.60 & + & Homo sapiens \\
\hline 2D6 & $2 \mathrm{F9Q}$ & 3.00 & - & Homo sapiens \\
\hline $2 \mathrm{R} 1$ & 2OJD & 2.80 & + & Homo sapiens \\
\hline $3 \mathrm{~A} 4$ & 1TQN & 2.05 & - & Homo sapiens \\
\hline $102 \mathrm{~A} 1$ & 1BU7 & 1.65 & - & $\begin{array}{l}\text { Bacillus megaterium (P450 } \\
\text { BM-3) }\end{array}$ \\
\hline $175 \mathrm{~A} 1$ & 1N97 & 1.80 & - & Thermus thermophilus \\
\hline \multicolumn{5}{|c|}{ Class I CYPs } \\
\hline $51 \mathrm{~B} 1$ & 1E9X & 2.10 & + & Mycobacterium tuberculosis \\
\hline $101 \mathrm{D}$ & 2CPP & 1.63 & + & $\begin{array}{l}\text { Pseudomonas putida } \\
\text { (P450cam) }\end{array}$ \\
\hline 107A1 & $10 X A$ & 2.10 & + & Saccharopolyspora erythrea \\
\hline 107L1 & 2BVJ & 2.10 & - & Streptomyces venezuelae \\
\hline $108 \mathrm{~A}$ & $1 \mathrm{CPT}$ & 2.30 & - & Pseudomonas sp. \\
\hline 119 & 1107 & 1.50 & - & Sulfolobus solfactaricus \\
\hline $154 \mathrm{~A} 1$ & $10 D O$ & 1.85 & + & Streptomyces coelicolor \\
\hline $154 C 1$ & $1 \mathrm{GWl}$ & 1.92 & - & Streptomyces coelicolor \\
\hline $158 \mathrm{~A} 1$ & $2 \mathrm{DKK}$ & 1.97 & + & Streptomyces coelicolor \\
\hline $158 \mathrm{~A} 2$ & $1 \mathrm{~S} 1 \mathrm{~F}$ & 1.50 & + & Streptomyces coelicolor \\
\hline $165 \mathrm{~B} 3$ & 1LFK & 1.70 & - & Amicolatopsis orientalis \\
\hline $165 C 4$ & 1UED & 1.90 & - & Amicolatopsis orientalis \\
\hline $167 A 1$ & 1Q5D & 1.93 & + & Polyangium cellulosum \\
\hline $176 \mathrm{~A} 1$ & $1 \mathrm{~T} 2 \mathrm{~B}$ & 1.70 & + & Citrobacter brakii \\
\hline 199A2 & $2 \mathrm{FR7}$ & 2.01 & - & $\begin{array}{l}\text { Rhodopseudomonas } \\
\text { palustris }\end{array}$ \\
\hline $245 \mathrm{~A} 1$ & 2Z3T & 1.90 & + & Streptomyces sp. TP-A0274 \\
\hline \multicolumn{5}{|c|}{ Other CYPs } \\
\hline $8 \mathrm{~A}$ & $2 \mid A G$ & 2.15 & - & Homo sapiens \\
\hline $55 \mathrm{~A} 2$ & 1CL6 & 1.70 & + & $\begin{array}{l}\text { Fusarium oxisporum (NO } \\
\text { reductase) }\end{array}$ \\
\hline $152 \mathrm{~A} 1$ & $1 I Z O$ & 2.10 & + & Bacillus subtilis ( ${\left.\mathrm{P} 450_{\mathrm{Bs} \beta}\right)}$ \\
\hline
\end{tabular}

Ligand-free and ligand-bound structures are indicated by - and +, respectively.

mammalian origin. The only exception is CYP102A1 (P450 BM-3) from Bacillus megaterium, which is a fusion enzyme, consisting of a P450 domain and a FMN/FAD reductase domain [15]. Because of its structural similarity to CYP102A1, the bacterial CYP175A1 isolated from the thermophilic Thermus thermophilus was also assigned to class II [14]. Additionally analyzed crystal structures were: CYP8A (human prostacyclin synthase), which accepts endoperoxides or hydroperoxides as substrates and does not require any electrontransfer partner or molecular oxygen [35]; CYP55A2 from Fusarium oxisporum and 152A1 from Bacillus subtilis $\left(\mathrm{P} 450_{\mathrm{Bs} \beta}\right)$ are representatives for CYPs which obtain electrons directly from $\mathrm{NAD}(\mathrm{P}) \mathrm{H}$ or catalyze a peroxide-dependent reaction. All structures represent the closed form of CYPs since including the open form as available for example for CYP2B4 [36] would worsen the alignment quality. Eleven recently published CYP structures were not included in the alignment but were used to validate the prediction of the structurally conserved regions.

\section{CYP Sequences}

The analysis of CYP sequences and structures was performed based on the updated version 2.02 of the CYPED [33]. It integrates sequences of 8614 proteins. The proteins are organized into 249 superfamilies and 619 homologous families according to Nelson [4]. Reliable multisequence alignments are available for each family. The sequences are annotated by automatically extracted GenBank annotations [37], which were manually enriched. Secondary structure information is available as DSSP annotation within the multisequence alignments for those homologous families containing members with existing PDB structures.

\section{Methods}

\section{Structure-based HMM profile}

SCRs were determined by the generation of a structurebased multisequence alignment using STAMP [38]. STAMP estimates the probability of structural equivalence of residues [39] and uses the Smith-Waterman algorithm [40] to determine the best path through a matrix of numerical pairwise similarity values of corresponding sequence positions. This allows STAMP to calculate two measures of alignment confidence: $\mathrm{P}_{\mathrm{ij}}{ }_{\mathrm{ij}}$, a measure for residue equivalence and $S_{c}$, the STAMP score, which reflects overall alignment quality. A $\mathrm{S}_{\mathrm{c}}>$ 5.5 implies a high degree of similarity of the considered structures. Stretches of residues in the alignment having $\mathrm{P}^{\prime}{ }_{\mathrm{ij}}>6.0$ imply regions of conserved secondary structure and are marked by black boxes in the alignment output. To visualize secondary structure information on the alignment output, STAMP uses DSSP [41] outputs. Therefore, in a first step DSSP was applied on the CYP structures to calculate secondary structure information. The resulting structure-based multisequence alignment was checked for correctly aligned secondary structures, ExxR motif and Cys-pocket. Regions with high $\mathrm{P}^{\prime}{ }_{\mathrm{ij}}$ which indicate conserved secondary structures were defined as SCR, extracted from the alignment and visualized (Figure 1) on the structure from CYP102A1 [PDB: 1BU7] as reference structure using PyMOL [42]. Structure-based HMM-profiles were derived from the structure-based multisequence alignments using HMMER http://hmmer.janelia.org/. 


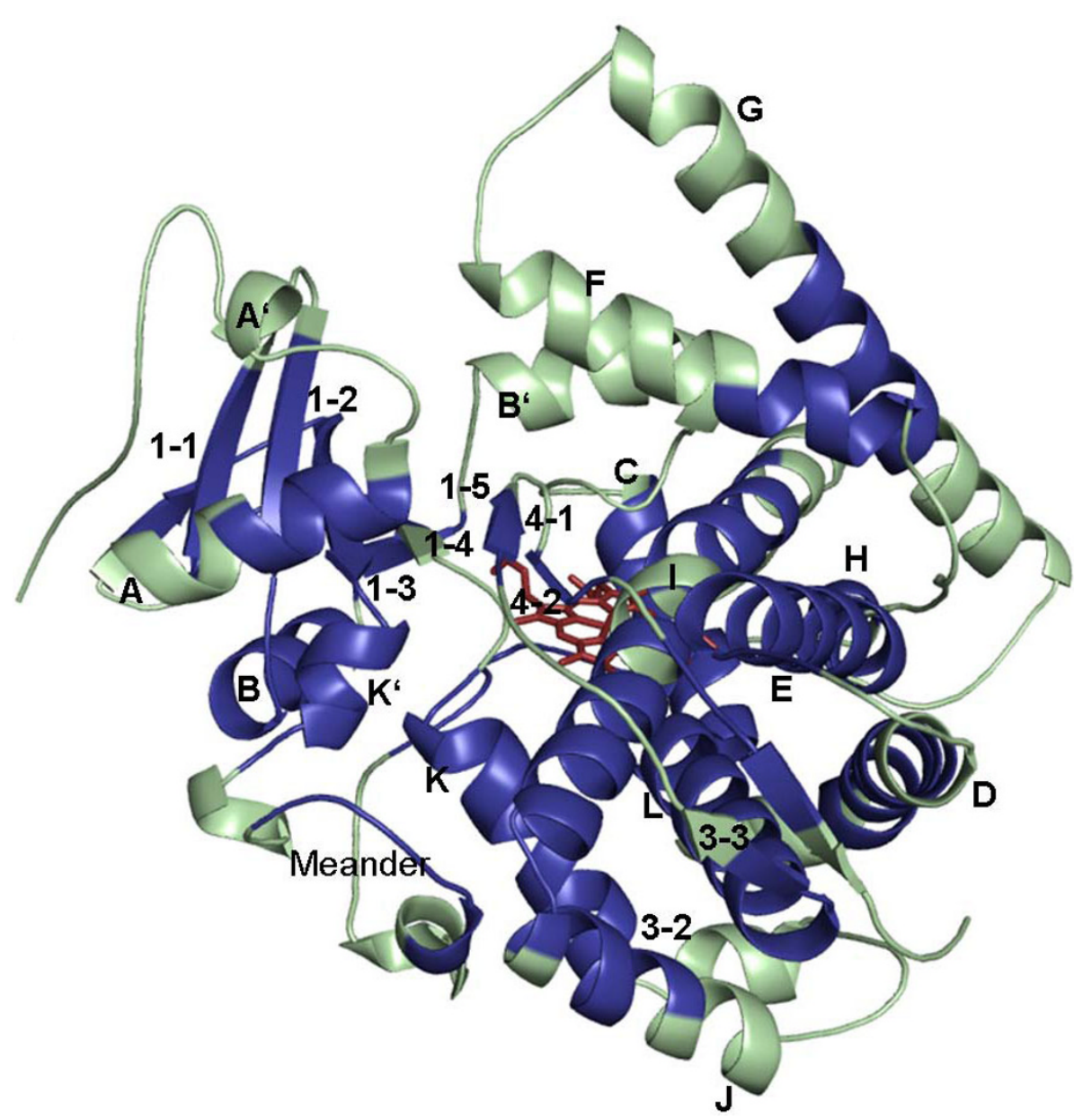

F.igure 1 SCRs of CYPs in a structural overview. The structurally conserved regions were derived from the STAMP alignment and mapped on the reference structure of P450 BM-3 from Bacillus megaterium [PDB: 1BU7]. The SCRs are highlighted in blue, whereas the variable regions are shown in green.

\section{Structural analysis}

Structural superpositions and visualizations were generated using PyMOL [42]. The analysis of the BC-loop region was performed by an overall superposition of all structures on CYP102A1 [PDB: 1BU7]. The visualization of the reductase interaction sites RIS1 and RIS2 was generated by the superposition of the FMN-domains of CYP102A1 [PDB: 1BVY] and the CPR-type FMN/FAD reductase from Rattus norvegicus [PDB: 3ES9] and the superposition of the P450-domains of CYP2C9 from Homo sapiens [PDB: 1OG2] CYP101D and from Pseudomonas putida [PDB: 2CPP] on the P450-domain of CYP102A1.

\section{Sequence analysis}

For the analysis of all CYP sequences, the CYPED and the $D W A R F$ system [43] were applied. The data warehouse system DWARF is the in-house repository for the CYPED data and assists local analysis. Besides integrating sequences and structures of this protein family, it provides a set of bioinformatics tools for sequence and structure analysis. We took advantage of its modular and extensible architecture and designed a Perl program which implements an automated procedure that subsequently generates a structure-based multisequence alignment for every CYPED entry by mapping it on the structure-based HMM profile which was derived from the STAMP alignment. Using the alignment row which represents the structure of CYP102A1 as a reference, the start and stop positions of each conserved secondary structure were identified within each alignment and transferred to the query sequence. Therefore, the absolute positions of the SCRs of each query sequence could be predicted. The positions were stored as annotations in the CYPED and are visualized in the multisequence alignments and on the feature page for each CYPED entry.

The same procedure as for the identification of the SCRs was applied to identify the specificity and regioselectivity 
determining position which corresponds to F87 in CYP102A1 in all sequences among the CYPED. Again, the sequence of the structure of CYP102A1 was used as the reference. Each CYPED query sequence was mapped on the structure-based HMM profile and the resulting alignment was used to determine the residue corresponding to F87.

The accuracy of this method was tested in a leave-oneout cross-validation [44] by generating for each of the 30 crystal structures a structure-based HMM profiles, leaving subsequently one structure out and mapping the sequence of the left-out crystal structure on the corresponding profile. The generated alignment was checked for the correct prediction of the residue corresponding to F87.

An online version of the prediction tool was integrated into the CYPED homepage. Since the method operates exclusively for sequences with CYP fold, input sequences are first checked for applicability by sequence homology via a BLAST [45] query using an E-value of $10^{-100}$. Structurally conserved regions are determined as described above.

\section{Results}

\section{Structural Core}

From the simultaneous superposition of the 31 structures using STAMP, a multiple sequence alignment could be derived which resulted in 257 structurally equivalent residues out of 400-450 residues. The calculated average RMS deviation after fitting all structures by these 257 residues was $2.4 \AA$ and their averaged sequence identity was $25 \%$. The overall STAMP alignment score $S_{c}$ was 6.0 and is above the threshold for highly similar structures. Stretches of structurally equivalent residues $\left(\mathrm{P}_{\mathrm{ij}}>\mathbf{6 . 0}\right)$ are marked by black boxes in the structure-based sequence alignment (figure S1, Additional file 1). The residues of the conserved core are organized into 19 SCRs that include at least partially all defined secondary structures $\alpha \mathrm{A}-\mathrm{L}$ and $\beta 1-4$. The SCRs extracted from the structural alignment were mapped on the reference structure CYP102A1 from Bacillus megaterium (Figure 1).

A topological overview of the conserved CYP structure illustrates the distribution of SCRs on the CYP structure (Figure 2). Some SCRs are part of individual secondary structures; other SCRs include several secondary structure elements. Among these, SCR3 comprises $\beta 1-2$ and $\alpha \mathrm{B}$, SCR7 $\beta 3-1$ and $\alpha \mathrm{E}$. SCR11 is assembled by $\alpha \mathrm{I}$ and $\alpha$ J and SCR13 by $\beta 1-4$ and $\beta 2-1 . \beta 2-2, \beta 1-3$ and $\alpha K^{\prime}$ together form SCR14 and the heme-binding Cys-pocket and $\alpha \mathrm{L}$ together form SCR16. The structural alignment further revealed that the $\beta-5$ sheet which is not present in all CYP structures does not belong to the conserved parts of the CYP structures [14]. The variable termini of the secondary structure elements $\alpha \mathrm{F}, \alpha \mathrm{G}, \alpha \mathrm{I}, \beta 1-4$, $\beta 4-1$, and the $B C$-loop are surrounding the heme and house the residues defining the SRS regions 1-6.

By applying the procedure on each CYPED sequence and mapping it on the HMM profile generated from the STAMP alignment, the SCRs could be identified and annotated in all sequence entries. The conserved secondary structures appear in the online version of the CYPED either within the annotated multisequence alignments or on the feature page of each protein entry. Its labelling appears in moving over the respective region. The results of the online prediction for any CYP sequence are displayed as colored and annotated regions and as a tabular output listing each conserved secondary structure and the corresponding start and stop position.

\section{BC-loop}

In CYP102A1, the phenylalanine at position 87 is assumed to mediate selectivity and activity. Due to its proximity to the heme center, this residue has a strong evidence to be involved in substrate binding and to control substrate specificity and regioselectivity [31]. Therefore, the identification of residues corresponding to this position would be beneficial in the design of CYPs with engineered properties. Since it is located in the SRS1 region of the highly variable $\mathrm{BC}$-loop the identification of this position in enzymes without structural information is not possible merely by sequence alignment. However, a comprehensive analysis of the $\mathrm{BC}$-loops in the structures analyzed in this work revealed that although being highly variable (Figure $3 \mathrm{~A}$ ), the $\mathrm{BC}$-loop in almost every structure of different proteins that were compared houses one residue, which points directly towards the heme, and remains rigid during substrate binding, which could be shown by comparing multiple structures of the same protein (figures S2 and S3, Additional file 1). By the overall superposition of structures of different proteins on the structure of CYP102A1, it could be shown that this position is located exactly at the same position, corresponding to the phenylalanine in CYP102A1 (Figure 3B) located at position 87 . Table 2 lists the corresponding residue in each structure.

To validate our structure-based method to assign SCRs in a one-leave-out cross-validation, the position which corresponds to F87 in CYP102A1 was predicted for each sequence of each structure. For 23 out of 30 $(80 \%)$, the predicted positions agreed with the crystal structure, in 7 CYPs they deviated by up to 2 residues. To further apply and to validate the procedure, the position was predicted in eleven structures published in progress of this study. 8 correct predictions, 2 deviations by one position, and one wrong prediction for the case of CYP7A1 which has in the crystal structure no residue located at this position, again confirmed an accuracy of $80 \%$. It should be noticed that in some crystal structures 


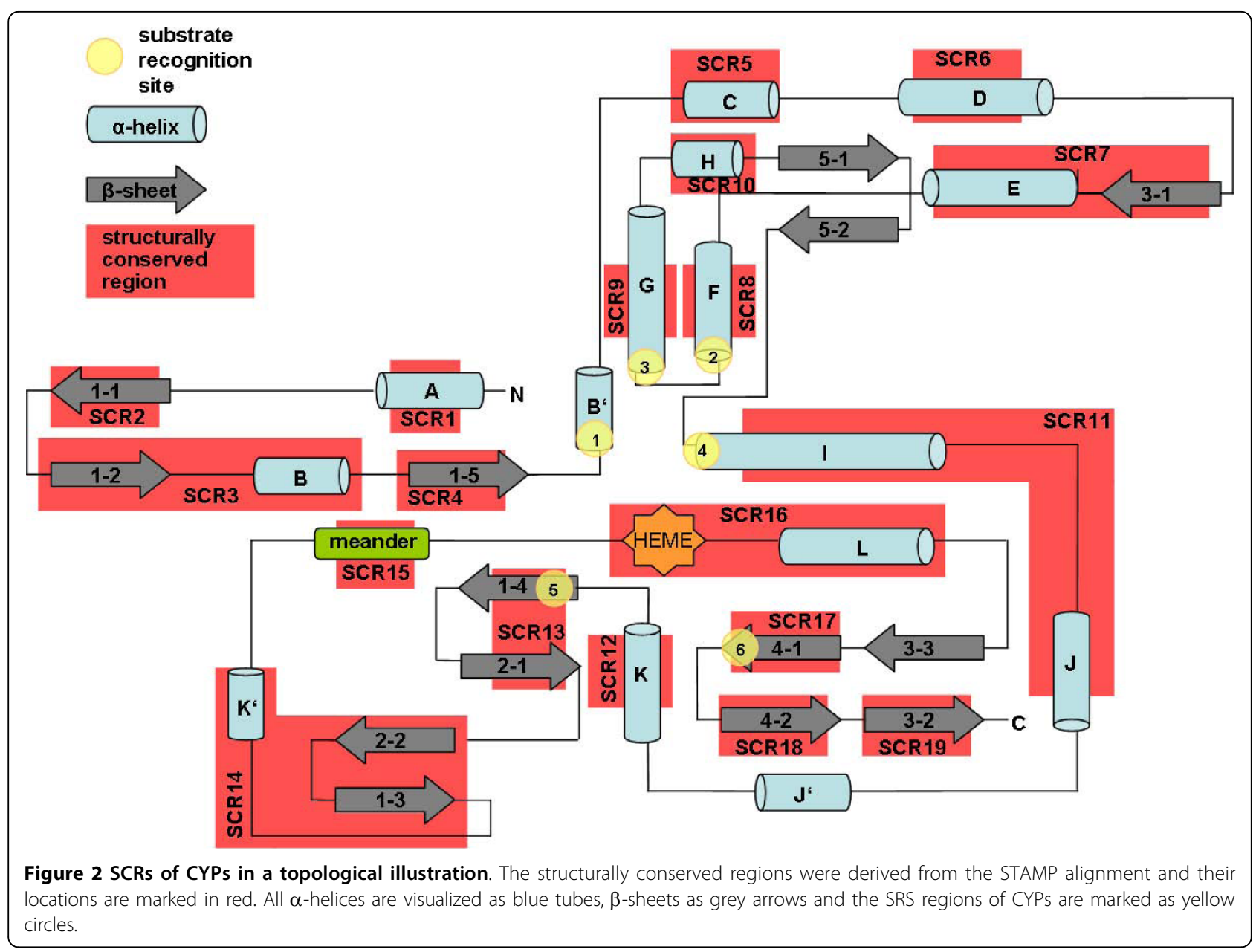

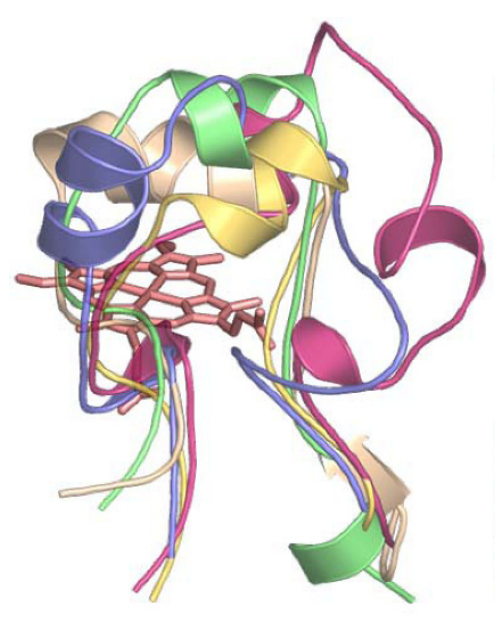

A

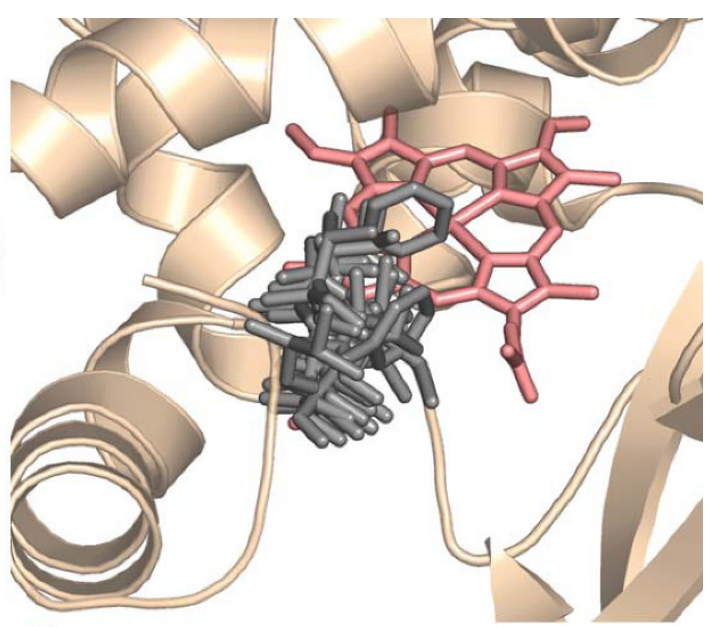

B

Figure 3 BC-loop region (SRS1) of CYPs. (A) Comparison of the BC-loops of P450 BM-3 [PDB: 1BU7] in beige, CYP2C9 [PDB: $10 \mathrm{OG}$ ] in green, CYP154C1 [PDB: 1GWl] in pink, CYP101D [PDB: 2CPP] in yellow and CYP107A1 [PDB: 1OXA] in blue. (B) BC-loop region in P450 BM-3 [PDB: 1BU7] and the position 87 corresponding residue in all 31 structures. 
Table 2 Positions which correspond to F87 in P450 BM-3 and predicted position, and prediction of positions in new structures

\begin{tabular}{|c|c|c|c|}
\hline$\overline{C Y P}$ & PDB-Code & $\begin{array}{l}\text { Position in crystal } \\
\text { structure }\end{array}$ & $\begin{array}{l}\text { Predicted } \\
\text { position }\end{array}$ \\
\hline $8 \mathrm{~A}$ & $2 \mid A G$ & - & - \\
\hline $51 \mathrm{~B} 1$ & 1E9X & - & V88 \\
\hline $55 \mathrm{~A} 2$ & $1 C L 6$ & V87 & V87 \\
\hline 101D & $2 \mathrm{CPP}$ & T101 & 199 \\
\hline $107 A 1$ & $10 X A$ & G91 & G91 \\
\hline 107L1 & 2BVJ & L93 & L93 \\
\hline $108 \mathrm{~A}$ & $1 \mathrm{CPT}$ & T103 & T103 \\
\hline 119 & 1107 & L69 & L69 \\
\hline $152 \mathrm{~A} 1$ & $11 Z O$ & Q85 & Q85 \\
\hline $154 \mathrm{~A} 1$ & $10 D O$ & F88 & F88 \\
\hline $154 \mathrm{C} 1$ & $1 \mathrm{GWl}$ & L93 & L93 \\
\hline $158 \mathrm{~A} 1$ & $2 \mathrm{DKK}$ & A97 & S95 \\
\hline $158 \mathrm{~A} 2$ & $1 \mathrm{~S} 1 \mathrm{~F}$ & G94 & G94 \\
\hline 165B3 & 1LFK & M89 & N87 \\
\hline $165 C 4$ & 1UED & 598 & 598 \\
\hline $167 A 1$ & 1Q5D & F96 & G94 \\
\hline $175 \mathrm{~A} 1$ & 1N97 & L80 & L80 \\
\hline $176 \mathrm{~A} 1$ & $1 \mathrm{~T} 2 \mathrm{~B}$ & A91 & M89 \\
\hline 199A2 & 2FR7 & L100 & L100 \\
\hline $245 \mathrm{~A} 1$ & $2 Z 3 T$ & V99 & V99 \\
\hline $1 \mathrm{~A} 2$ & $2 \mathrm{HI} 4$ & T124 & $\mathrm{S} 126$ \\
\hline $2 A 6$ & $1 Z 10$ & V117 & V117 \\
\hline $2 \mathrm{~A} 13$ & $2 \mathrm{P} 85$ & A117 & A117 \\
\hline $2 \mathrm{~B} 4$ & 1SUO & 1114 & 1114 \\
\hline $2 C 5$ & $1 N 6 B$ & A113 & A113 \\
\hline $2 C 8$ & 1PQ2 & 1113 & 1113 \\
\hline $2 C 9$ & $10 G 2$ & V113 & V113 \\
\hline 2D6 & $2 \mathrm{F9Q}$ & F120 & F120 \\
\hline $2 \mathrm{R} 1$ & 2OJD & L125 & L125 \\
\hline $3 \mathrm{~A} 4$ & $1 \mathrm{TQN}$ & $\mathrm{S} 119$ & S119 \\
\hline $102 \mathrm{~A} 1$ & $\begin{array}{l}\text { 1BU7 } \\
\text { (reference) }\end{array}$ & F87 & F87 \\
\hline $2 \mathrm{E} 1$ & $3 \mathrm{E} 4 \mathrm{E}$ & 194 & 194 \\
\hline 3A43 & $2 \mathrm{VOM}$ & S119 & S119 \\
\hline 7A1 & $2 \mathrm{DAX}$ & - & D98 \\
\hline 19A1 & $3 \mathrm{EQM}$ & F134 & F134 \\
\hline $46 \mathrm{~A} 1$ & 2Q9F & V126 & S127 \\
\hline $74 \mathrm{~A} 1$ & $2 \mathrm{RCH}$ & $\mathrm{S} 128$ & L127 \\
\hline $105 \mathrm{~A} 1$ & $2 Z B X$ & 196 & 196 \\
\hline $105 \mathrm{~K} 1$ & $2 Z 36$ & L96 & L96 \\
\hline $120 \mathrm{~A} 1$ & 2VE3 & A94 & A94 \\
\hline $231 \mathrm{~A} 2$ & $2 \mathrm{RFB}$ & 148 & 148 \\
\hline $248 \mathrm{~A}$ & 3BUJ & L80 & L80 \\
\hline
\end{tabular}

the residue numbering of the structures deviates from the residue numbering in the sequences due to missing residues and therefore the numbering of the protein structure was considered. The crystal structure of
CYP231A from the thermoacidophilic Picrophilus torridus was missing a part of the BC-loop [46] which made the prediction not clearly defined.

\section{Amino acid composition of the F87 corresponding position}

In addition to the identification of the F87 corresponding position, a comprehensive analysis of the sequences of all 8614 CYPED protein entries was performed in respect to the amino acid composition, by a prediction of the position in all sequences analogous to the SCR prediction. It could be observed that $73 \%$ of the residues predicted at this position include aliphatic residues and phenylalanine. The remaining $24 \%$ at this position are small polar residues and only are $3 \%$ charged residues. Phenylalanine (22\%), leucine (22\%), and valine (12\%) were the most frequently occuring amino acids followed by isoleucine $(10 \%)$ and alanine $(9 \%)$. Other amino acids appear more rarely with frequencies less than $4 \%$ (Figure 4 ). A predicted gap at this position indicates that the $\mathrm{BC}$-loop region houses no residue which is located close to the heme or the BC-loop itself winds away from the active site as it could be observed for example in the structures CYP8A and CYP51B1.

\section{Analysis of reductase interaction sites}

The structural regions $\alpha \mathrm{J} / \mathrm{J}^{\prime}$ and the insertion between the meander loop and the Cys-pocket are of particular interest since they were previously proposed to form the reductase interacting face of the molecules [6]. These sites strongly vary in their length and conformation. The structural analysis (Figure 5) reflects the differences of $\alpha \mathrm{J} / \mathrm{J}$ ' (further referred to as reductase interaction site 1, RIS1) (Figure 5A) and the insertion between meander loop and Cys-pocket (further referred to as reductase interaction site 2, RIS2) (Figure 5B) of CYPs from different redox classes. A comparison of the human CYP2C9 and the bacterial P450cam CYP101D shows that RIS1 $\left(\alpha J / J^{\prime}\right.$ region) of CYP2C 9 is 18 residues longer. RIS2 differs by 9 residues between CYP2C9 and CYP101D. By counting the number of residues spanning these regions in the STAMP alignment (figure S1, Additional file 1), it was revealed that these regions in class II CYPs interacting with CPR-type reductases are long, in class I CYPs extremely short or not existing at all and that those CYPs which do not require any electron transfer partner form a subgroup of class II, in some cases with extremely long loops. The $\alpha \mathrm{J} / \mathrm{J}^{\prime}$ region differs from 21 to 22 residues for class II (long) and 3 to 5 residues for class I CYPs (short). The length of the meander insertion differs from 11 to 17 residues for class II (long), up to 23 residues (very long) in those CYPs which do not require a redox partner and 3 to 5 residues for class I CYPs (short). 


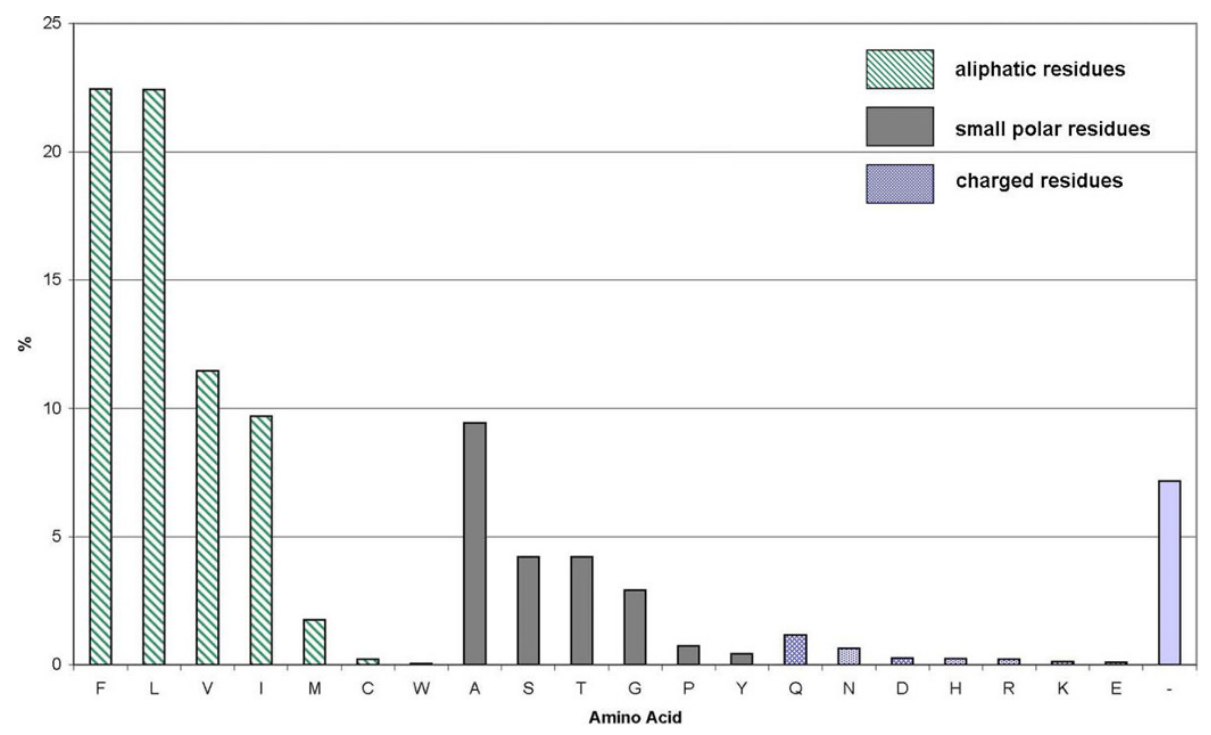

Figure 4 Residues at F87 corresponding position. Amino acid composition of predicted F87 corresponding positions in all 8614 CYPED proteins. Green bars correspond to the percentage of aliphatic residues and phenylalanine, grey bars to amino acids of small polar nature and blue bars to charged amino acids. '-' denotes a gap.

Counting the number of amino acids in each CYPED sequence for RIS1 (Figure 6A) revealed two peaks in the RIS1 length distribution. This allowed defining two classes. Proteins having short RIS1 with less than 10 residues spanning the $\alpha \mathrm{J} / \mathrm{J}^{\prime}$ region make up $17.5 \%$ of all protein entries. According to the result of the length analysis of RIS1 of the structural alignment, they comprise class I CYPs. Proteins having long RIS1 with more than 15 residues spanning the $\alpha$ J/J' region make up $81 \%$ of all protein entries. According to the result of the length analysis of RIS1 of the structural alignment, they comprise class II CYPs. Only $1 \%$ of all protein entries can not reliably be assigned by RIS1 length since their length is in between 10 and 15 amino acids.

The analysis of the length of RIS2 in each CYPED sequence (Figure 6B) showed a distribution in three main areas. Therefore, three classes according to the result of the length analysis of RIS2 in the structural alignment were defined. Proteins having short RIS2 with less than 7 residues spanning the meander insertion
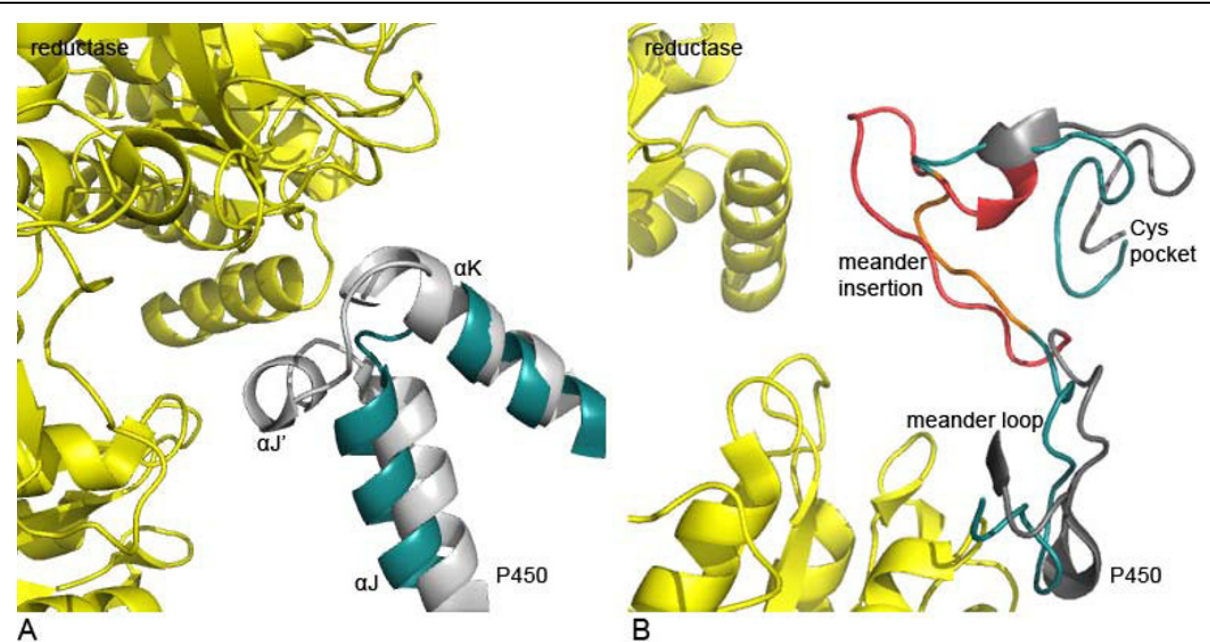

Figure 5 Sites interacting with potential redox partners. The CPR-type FMN/FAD from Rattus norvegicus [PDB: 3ES9] is shown in yellow, the parts of the P450 domain of CYP2C9 from Homo sapiens [PDB: 1OG2] are shown in grey and green for CYP101D from Pseudomonas putida [PDB: 2CPP], respectively. (A) Comparison RIS1 ( $\alpha \mathrm{J} / \mathrm{J}^{\prime}$ region) of the human CYP2C9 and P450cam CYP101D. (B) Comparison of RIS2 (meander insertion) of the human CYP2C9 and P450cam CYP101D. 

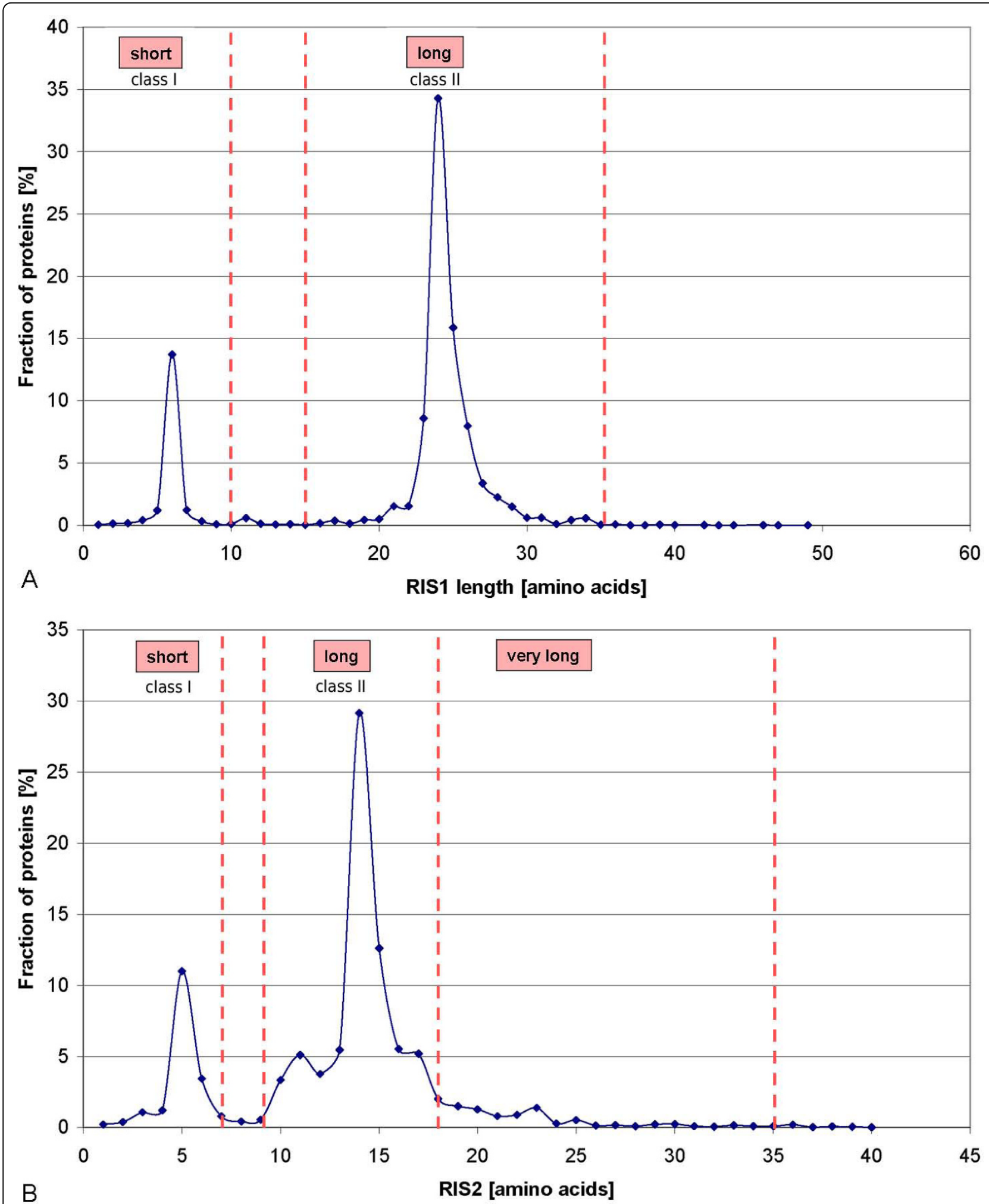

Figure 6 Analysis of RIS length. (A) Fraction of proteins for each RIS1 ( $\alpha \mathrm{J} / J^{\prime}$ region) length. (B) Fraction of proteins for each RIS2 (meander insertion) length. 
make up $18 \%$ of all protein entries. According to the result of the length analysis of RIS2 in the structural alignment, they comprise class I CYPs. Proteins having long RIS2 with between 11 and 17 residues spanning the meander insertion make up $66 \%$ of all protein entries. According to the result of the length analysis of RIS2 in the structural alignment, they comprise class II CYPs, with a subgroup of proteins having very long RIS2 with more than 18 residues spanning the meander insertion. $4 \%$ of all protein entries can not reliably be assigned by RIS2 length since their length is in between 8 and 10 amino acids.

$0.5 \%$ of entries with RIS1 and $0.5 \%$ of entries with RIS2 length above 35 amino acids were formally assigned to class II, but could not be further analyzed since they comprise biochemically not characterized proteins.

\section{Discussion}

Despite their inherently low sequence similarity, all CYPs share a common structural fold. The well-defined secondary structure elements can be found in all determined crystal structures, which house their active-site with the cofactor heme deeply inside the protein [21]. The generation of a structural alignment out of 31 CYP structures revealed structurally conserved regions which contain most of the described secondary structure elements of the CYP fold. It could be shown that some of the secondary structure elements merge together to structure modules, described as structurally conserved regions (SCR) $1-19$, reflecting the modular structure of cytochrome P450 monooxygenases. The generation of a reliable structure-based HMM profile which was applied to every CYPED entry assisted in consistently annotating the conserved secondary structures in the CYPED entries. But besides addressing the problem of predicting conserved regions, an even more challenging issue could be solved: the identification and classification of the variable regions.

Since the residues that determine the substrate specificity of CYPs are assumed to lie in the variable regions $[8,22]$, their identification is of greatest interest for engineering of biochemical properties. Two of the six proposed substrate recognition sites, SRS1 and SRS5, together with the helix I directly flank the substrate binding cavity and are therefore supposed to interact with the substrate [27]. SRS1 houses a residue, which previously was described as essential for activity, regio- and stereoselectivity in CYP102A1 [28-30]. Located at position 87 and pointing directly towards the heme, a corresponding residue to this phenylalanine can be found in almost all CYP structures. Its location in the highly variable $\mathrm{BC}$-loop region makes its determination very difficult in sequences without structural information.
The position, which corresponds to F87 in CYP102A1 could be correctly predicted in almost $80 \%$ of all analyzed CYP structures. By surveying more recent CYP structures, the validity of the prediction could be confirmed. The analysis of this position in all 8614 CYP sequences in the CYPED revealed that the residues at this position predominantly are of aliphatic nature or a phenylalanine, less frequently small polar amino acids and only very infrequently of charged nature. Since the characteristics of the residue at this position highly influence substrate specificity and regioselectivity, its identification contributes to the design of CYPs with more suitable properties for biocatalytic applications.

Even though there were two reductase interaction sites proposed to be located in $\alpha \mathrm{J} / \alpha \mathrm{J}$ ' and in the insertion following the meander loop [6], termed RIS1 and RIS2, these regions which are highly variable in sequence and structure were difficult to determine in sequences. The identification of the preceding and the successive SCR solved this problem. Depending on the length for RIS1, two classes (short and long RIS1) and three classes for RIS2 (short, long and very long RIS2) were introduced. From the analysis of the CYP structures in respect to their redox partner it was assumed that class II CYPs have long RIS1 and long RIS2, class I CYPs have short RIS1 and short RIS 2.

The largest percentage of all CYPs has long RIS1 and long RIS2 (53\%). All CYPs with available structure which possess these long loops clearly belong to class II, and most of them are of human origin. The class II protein P450 BM-3 also shows the characteristic CPR-interacting loop length. The $12 \%$ of proteins with short RIS1 and RIS2, respectively, are assumed to be class I proteins. $27 \%$ could not be clearly classified, either because of unusual long loops (above 35 residues), or a combination of short RIS1 with long RIS2 and vice versa. This comparison of reductase interaction sites allows drawing conclusions on its reductase interaction.

The remaining $8 \%$ of CYPs consist of proteins with long RIS1 and very long RIS2. Members of this unusual group cannot easily be categorized in regards to their reductase interaction. For example, the human prostacyclin synthase CYP8A1, which has endoperoxidase activity and does not require a reductase as source of electrons, is a representative of this class of proteins [35]. It has a long RIS1, consisting of 22 amino acids and a very long RIS2 of 23 amino acids. However, the crystal structure for the human cholesterol 7 alphahydroxylase CYP7A1 which was recently solved also contains very long proximal loops [47] that were correctly predicted containing 22 (RIS1) and 23 (RIS2) amino acids. CYP7A1 was previously compared to the structure of CYP8A1 [48], but in contrast is a typical monooxygenase. 
The fatty acid hydroxylase CYP152A1 from Bacillus subtilis $\left(\mathrm{P} 450_{\mathrm{Bs} \beta}\right)$ is a hydrogen peroxide driven enzyme [49] and therefore could be assigned to those CYPs which do not require a redox partner. CYP152A1 has a short RIS1 of 5 amino acid residues and a long RIS2 of 11 residues, like the CPR-type interacting class II CYPs, which is unexpected for this kind of CYP. Indeed, CYP152A1 and its homologous protein CYP152A2 from Clostridium acetobutylicum ( $\mathrm{P} 450_{\mathrm{CLA}}$ ) experimentally showed much higher conversions in the presence of a CPR-type reductase than in the presence of hydrogen peroxide and the absence of a reductase [50]. The recently solved crystal structure of the allene oxide synthase CYP74A1 is an atypical cytochrome P450 family member and does not require a reductase [51]. However, CYP74A1 also shows similar loop lengths to class II CYPs of RIS1 of 21 AS and RIS2 of 10 AS. Due to an unusual nine amino acid insert in the Cys-pocket which allows its access to the protein surface, the interaction with a redox partner might be disrupted [51]. Therefore, CYP74A1 cannot be compared to typical monooxygenases with similar RIS1 and RIS2 length by our model.

Since most CYPs require electrons from a redox partner, and CYP152A1 and CYP152A2 showed higher activities by adding a reductase, it can be assumed that the interaction of CYPs with reductases plays a pivotal role in the CYP mechanism. Finding the optimal redox partner for CYPs may significantly enhance their activity but is quite difficult. The analysis and classification which led to the prediction of possible redox partner interactions offers the potential of engineering enhanced interactions.

\section{Conclusion}

In order to navigate in CYP sequences and to determine functionally relevant residues, a procedure which allows identifying conserved modules and functionally relevant sites within variable regions was implemented. Regions involved in substrate binding as well as redox partner recognition and interaction could be determined in the absence of structural information, based on sequence only. The structurally annotated sequences and multisequence alignments are accessible on the current version of the CYPED http://www.cyped.uni-stuttgart.de. Via a web interface integrated in the CYPED homepage at http://www.cyped.uni-stuttgart.de/cgi-bin/strpred/ dosecpred.pl, the structural prediction is provided for every sequence which is similar to CYPs or presumably shares the CYP fold. The navigation in CYP sequences and the determination of functionally relevant sites in turn is a great advantage in the prediction of promising targets for the design of CYPs with improved biocatalytic properties.

\section{Additional material}

Additional file 1: This file contains figures S1, S2, and S3 mentioned in the text.

\section{List of abbreviations}

CYP: Cytochrome P450 monooxygenase; CYPED: Cytochrome P450 Engineering Database; CPR: Cytochrome P450 reductase; P450 BM-3: Cytochrome P450 monooxygenase BM-3 from Bacillus megaterium; DWARF: Data Warehouse for Analyzing Protein Families; BLAST: Basic Local Alignment Search Tool; HMM:Hidden Markov model; STAMP: Structural Alignment of Multiple Proteins; DSSP: Define Secondary Structure of Proteins; SRS: Substrate recognition site; SCR: Structurally conserved region; RIS: Reductase interaction site.

\section{Acknowledgements}

This work was supported by the German Research Foundation (SFB706).

\section{Authors' contributions}

DS implemented the program, performed the analysis and wrote the manuscript. MW contributed to the analysis and to the manuscript. FW carried out the annotation and generated the web interface. JP supervised the project and finalized the manuscript. All authors read and approved the final version of the manuscript.

Received: 28 January 2010 Accepted: 15 October 2010 Published: 15 October 2010

\section{References}

1. Ortiz de Montellano PR: Cytochrome P450: structure, mechanism and biochemistry. New York, Plenum Press 1995.

2. Raucy $\mathrm{J}$, Allen SW: Recent advances in P450 research. Pharmacogenomics J 2001, 1:178-186.

3. Urlacher VB, Eiben S: Cytochrome P450 monooxygenases: perspectives for synthetic application. Trends Biotechnol 2006, 24:324-330.

4. Nelson DR: Cytochrome P450 nomenclature, 2004. Methods Mol Biol 2006, 320:1-10.

5. Graham SE, Peterson JA: How similar are P450s and what can their differences teach us? Arch Biochem Biophys 1999, 369:24-29.

6. Hasemann CA, Kurumbail RG, Boddupalli SS, Peterson JA, Deisenhofer J: Structure and function of cytochromes P450: a comparative analysis of three crystal structures. Structure 1995, 3:41-62.

7. de Graaf C, Vermeulen NPE, Feenstra KA: Cytochrome p450 in silico: an integrative modeling approach. J Med Chem 2005, 48:2725-2755.

8. Peterson JA, Graham SE: A close family resemblance: the importance of structure in understanding cytochromes P450. Structure 1998, 6:1079-1085.

9. Werck-Reichhart D, Feyereisen R: Cytochromes P450: a success story. Genome Biol 2000, 1:REVIEWS3003.

10. Hannemann F, Bichet A, Ewen KM, Bernhardt R: Cytochrome P450 systems-biological variations of electron transport chains. Biochim Biophys Acta 2007, 1770:330-344.

11. McLean KJ, Sabri M, Marshall KR, Lawson RJ, Lewis DG, Clift D, Balding PR, Dunford AJ, Warman AJ, McVey JP, et al: Biodiversity of cytochrome P450 redox systems. Biochem Soc Trans 2005, 33:796-801.

12. Munro AW, Girvan HM, McLean KJ: Cytochrome P450-redox partner fusion enzymes. Biochim Biophys Acta 2007, 1770:345-359.

13. Roberts GA, Grogan G, Greter A, Flitsch SL, Turner NJ: Identification of a new class of cytochrome P450 from a Rhodococcus sp. Journal of Bacteriology 2002, 184:3898-3908.

14. Baudry J, Rupasinghe S, Schuler MA: Class-dependent sequence alignment strategy improves the structural and functional modeling of P450s. Protein Eng Des Sel 2006, 19:345-353.

15. Munro AW, Leys DG, McLean KJ, Marshall KR, Ost TWB, Daff S, Miles CS, Chapman SK, Lysek DA, Moser CC, et al: P450 BM3: the very model of a modern flavocytochrome. Trends Biochem Sci 2002, 27:250-257. 
16. Bernhardt R: Cytochrome P450: structure, function, and generation of reactive oxygen species. Rev Physiol Biochem Pharmacol 1996, 127:137-221.

17. Wade RC, Motiejunas D, Schleinkofer K, Sudarko, Winn PJ, Banerjee A, Kariakin A, Jung C: Multiple molecular recognition mechanisms. Cytochrome P450-a case study. Biochim Biophys Acta 2005, 1754:239-244.

18. Guengerich FP, Johnson WW: Kinetics of ferric cytochrome P450 reduction by $\mathrm{NADPH}-$ cytochrome $\mathrm{P} 450$ reductase: Rapid reduction in the absence of substrate and variations among cytochrome P450 systems. Biochemistry 1997, 36:14741-14750.

19. Guengerich FP: Rate-limiting steps in cytochrome P450 catalysis. Biol Chem 2002, 383:1553-1564

20. Bernhardt R: Cytochromes P450 as versatile biocatalysts. J Biotechnol 2006, 124:128-145.

21. Mestres J: Structure conservation in cytochromes P450. Proteins 2005, 58:596-609.

22. Gotoh O: Substrate recognition sites in cytochrome P450 family 2 (CYP2) proteins inferred from comparative analyses of amino acid and coding nucleotide sequences. J Biol Chem 1992, 267:83-90.

23. Li HY, Poulos TL: The structure of the cytochrome p450BM-3 haem domain complexed with the fatty acid substrate, palmitoleic acid. Nature Structural Biology 1997, 4:140-146.

24. Li HY, Poulos TL: Fatty acid metabolism, conformational change, and electron transfer in cytochrome P-450(BM-3). Biochimica Et Biophysica Acta-Molecular and Cell Biology of Lipids 1999, 1441:141-149.

25. Branco RJF, Seifert A, Budde M, Urlacher VB, Ramos MJ, Pleiss J: Anchoring effects in a wide binding pocket: The molecular basis of regioselectivity in engineered cytochrome P450 monooxygenase from B. megaterium. Proteins-Structure Function and Bioinformatics 2008, 73:597-607.

26. Seifert A, Tatzel S, Schmid RD, Pleiss J: Multiple molecular dynamics simulations of human P450 monooxygenase CYP2C9: The molecular basis of substrate binding and regioselectivity toward warfarin. ProteinsStructure Function and Bioinformatics 2006, 64:147-155.

27. Seifert A, Pleiss J: Identification of selectivity-determining residues in cytochrome P450 monooxygenases: a systematic analysis of the substrate recognition site 5. Proteins 2009, 74:1028-1035

28. Li HM, Mei LH, Urlacher VB, Schmid RD: Cytochrome P450 BM-3 evolved by random and saturation mutagenesis as an effective indolehydroxylating catalyst. Appl Biochem Biotechnol 2008, 144:27-36.

29. Urlacher V, Schmid RD: Biotransformations using prokaryotic P450 monooxygenases. Curr Opin Biotechnol 2002, 13:557-564.

30. Urlacher VB, Makhsumkhanov A, Schmid RD: Biotransformation of betaionone by engineered cytochrome P450 BM-3. Appl Microbiol Biotechnol 2006, 70:53-59.

31. Seifert A, Vomund S, Grohmann K, Kriening S, Urlacher VB, Laschat S, Pleiss J: Rational design of a minimal and highly enriched CYP102A1 mutant library with improved regio-, stereo- and chemoselectivity. Chembiochem 2009, 10:853-861.

32. Fischer M, Knoll M, Sirim D, Wagner F, Funke S, Pleiss J: The Cytochrome P450 Engineering Database: a navigation and prediction tool for the cytochrome P450 protein family. Bioinformatics 2007, 23:2015-2017.

33. Sirim D, Wagner F, Lisitsa A, Pleiss J: The cytochrome P450 engineering database: Integration of biochemical properties. BMC Biochem 2009, $10: 27$

34. Berman HM, Battistuz T, Bhat TN, Bluhm WF, Bourne PE, Burkhardt $\mathrm{K}$, Feng Z, Gilliland GL, lype L, Jain S, et al: The Protein Data Bank. Acta Crystallogr D Biol Crystallogr 2002, 58:899-907.

35. Chiang C, Yeh H, Wang L, Chan N: Crystal Structure of the Human Prostacyclin Synthase. J Mol Biol 2006, 364:266-274.

36. Scott EE, He YA, Wester MR, White MA, Chin CC, Halpert JR, Johnson EF, Stout CD: An open conformation of mammalian cytochrome P4502B4 at 1.6-angstrom resolution. Proceedings of the National Academy of Sciences of the United States of America 2003, 100:13196-13201.

37. Benson DA, Karsch-Mizrachi I, Lipman DJ, Ostell J, Wheeler DL: GenBank. Nucleic Acids Res 2008, 36:D25-D30.

38. Russell RB, Barton GJ: Multiple protein sequence alignment from tertiary structure comparison: assignment of global and residue confidence levels. Proteins 1992, 14:309-323.

39. Rossmann MG, Argos P: Exploring structural homology of proteins. J Mol Biol 1976, 105:75-95.

40. Smith TF, Waterman MS: Identification of common molecular subsequences. J Mol Biol 1981, 147:195-197.
41. Kabsch W, Sander C: Dictionary of protein secondary structure: pattern recognition of hydrogen-bonded and geometrical features. Biopolymers 1983, 22:2577-2637.

42. Delano WL: The PyMOL Molecular Graphics System. San Carlos, CA, USA: Delano Scientific 2002.

43. Fischer M, Thai QK, Grieb M, Pleiss J: DWARF-a data warehouse system for analyzing protein families. BMC Bioinformatics 2006, 7:495.

44. Picard RR, Cook RD: Cross-Validation of Regression Models. Journal of the American Statistical Association 1984, 79:575-583.

45. Altschul SF, Madden TL, Schaeffer AA, Zhang J, Zhang Z, Miller W, Lipman DJ: Gapped BLAST and PSI-BLAST: a new generation of protein database search programs. Nucleic Acids Res 1997, 25:3389-3402.

46. Ho WW, Li H, Nishida CR, de Montellano PRO, Poulos TL: Crystal structure and properties of CYP231A2 from the thermoacidophilic archaeon Picrophilus torridus. Biochemistry 2008, 47:2071-2079.

47. Strushkevich NV, Tempel W, Dombrovski L, Dong A, Loppnau P, Arrowsmith $\mathrm{CH}$, Edwards AM, Bountra C, Wilkstrom M, Bochkarev A, Park H: Crystal structure of human CYP7A1. To be Published .

48. Mast N, Graham SE, Andersson U, Bjorkhem I, Hill C, Peterson J, Pikuleva IA: Cholesterol binding to cytochrome P450 7A1, a key enzyme in bile acid biosynthesis. Biochemistry 2005, 44:3259-3271.

49. Lee DS, Yamada A, Sugimoto H, Matsunaga I, Ogura H, Ichihara K Adachi SI, Park SY, Shiro Y: Substrate recognition and molecular mechanism of fatty acid hydroxylation by cytochrome P450 from Bacillus subtilis. Crystallographic, spectroscopic, and mutational studies. J Biol Chem 2003, 278:9761-9767.

50. Girhard M, Schuster S, Dietrich M, Dürre P, Urlacher VB: Cytochrome P450 monooxygenase from Clostridium acetobutylicum: a new alpha-fatty acid hydroxylase. Biochem Biophys Res Commun 2007, 362:114-119.

51. Lee DS, Nioche P, Hamberg M, Raman CS: Structural insights into the evolutionary paths of oxylipin biosynthetic enzymes. Nature 2008, 455:363-U327.

doi:10.1186/1472-6807-10-34

Cite this article as: Sirim et al:: Prediction and analysis of the modular structure of cytochrome P450 monooxygenases. BMC Structural Biology 2010 10:34.

\section{Submit your next manuscript to BioMed Central and take full advantage of:}

- Convenient online submission

- Thorough peer review

- No space constraints or color figure charges

- Immediate publication on acceptance

- Inclusion in PubMed, CAS, Scopus and Google Scholar

- Research which is freely available for redistribution

Submit your manuscript at www.biomedcentral.com/submit
C Biomed Central 